\title{
HEMATOME ORGANISE DU SINUS MAXILLAIRE A PROPOS D'UN CAS
}

\author{
N . BELTAIEF , S . TEMIMI , S . TRABELSI , I . DRIDI , R . ZAININE , K . NOUIRA* , S. KHARRAT , \\ S. SAHTOUT, A. CHARFI, E . MNIF* , G. BESBES. \\ SERVICE ORL ET CHIRURGIE MAXILLOFACIALE \\ *SERVICE DE RADIOLOGIE \\ HOPITAL LA RABTA. TUNIS. TUNISIE
}

\begin{abstract}
L'hématome organisé du sinus maxillaire est une entité rare. Son étiopathogénie reste incertaine. Nous rapportons le cas d'une patiente âgée de 16 ans présentant une épistaxis unilatérale droite de grande abondance avec une endoscopie nasale strictement normale. L'imagerie était en faveur d'une tumeur vasculaire du sinus maxillaire. Une exérèse chirurgicale complète a été réalisée par voie combinée avec des suites simples. L'examen anatomopathologique a conclu à un hématome organisé du sinus maxillaire.

Nous discutons, dans ce travail, l'étiopathogénie, les caractéristiques cliniques, radiologiques et les volets thérapeutiques de cette entité.
\end{abstract}

Mots clés : Hématome organisé, sinus maxillaire, tomodensitométrie, chirurgie.

\section{SUMMARY}

Organized hematoma of the maxillary sinus is a rare pathology. We report a case of a 16 year-old female who developed recurrent unilateral epistaxis with normal nasal endoscopy. The tomodensitometry and MRI showed expansive vascular mass in the maxillary sinus. A surgical resection with combined approach was performed. Histology showed organized hematoma of the maxillary sinus. This paper is discussing ethiopathogeny, clinical and radiologic features and therapy of this pathology.

Keywords : Organized hematoma, maxillary sinus, tomodensitometry, surgery.

\section{INTRODUCTION}

Les hématomes du sinus maxillaire se résorbent habituellement sans séquelles et de façon complète. Ces hématomes peuvent devenir, exceptionnellement, encapsulés formant une tumeur kystique : c'est l'hématome organisé du sinus maxillaire (HOSM). Cette entité rare et bénigne peut simuler une tumeur maligne du sinus maxillaire par son pouvoir lytique sur les structures adjacentes. Nous rapportons un cas d' HOSM chez une jeune patiente qui a bénéficié d'une exérèse chirurgicale au service d'ORL et CMF la Rabta, tout en rappelant les caractéristiques étiopathogéniques, cliniques, paracliniques et thérapeutiques de cette affection.

\section{OBSERVATION}

II s'agit d'une patiente âgée de 16 ans, aux antécédents d'épistaxis unilatérale droite récidivante de faible abondance depuis 10 ans non explorée. Elle a été hospitalisée dans notre service en urgence pour épistaxis unilatérale droite de grande abondance avec retentissement sur l'état hémodynamique. Par ailleurs, elle se plaint depuis 6 mois d'algies faciales en regard du sinus maxillaire droit sans obstruction nasale ni rhinorrhée ou anosmie. Pas de notion de traumatisme ni de trouble de l'hémostase.

A l'endoscopie nasale, la muqueuse nasale était inflammatoire, les méats moyens et le cavum étaient libres.
Le reste de l'examen était sans particularités. A la biologie l'hémoglobine était de $6,7 \mathrm{~g} / \mathrm{dl}$; les plaquettes étaient de 152000 éléments/mm3. Le bilan d'hémostase était correct.

Le scanner du massif facial, demandé en urgence devant des algies faciales et une épistaxis de grande abondance récidivantes, a objectivé une formation tissulaire hétérogène intrasinusienne droite de $18 \mathrm{~mm}$, spontanément isodense sans visualisation de calcifications se rehaussant intensément dès le temps artériel avec des structures vasculaires superficielles et entourée d'une hyperplasie muqueuse (figure 1).
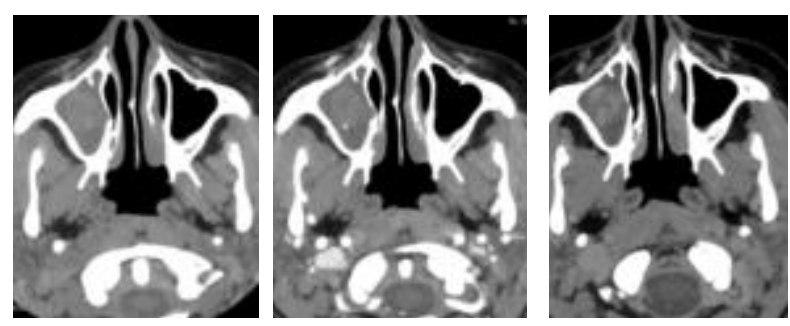

Fig. 1: TDM du massif facial en coupes axiales, sans (a) et avec injection de produit de contraste, temps précoce artériel (b) et tardif (c): Comblement total du sinus maxillaire droit centré par une fomation tissulaire spontanément isodense prenant le PDC dès le temps artériel et son rehaussement s'intensifie sur les coupes tardives. 
L'IRM du massif facial a montré la même formation du sinus maxillaire droit, bien limitée, de signal hétérogène, siège de quelques foyers en hypersignal T1 spontané et rehaussée après injection de Gadolinium (figure 2,3,4).
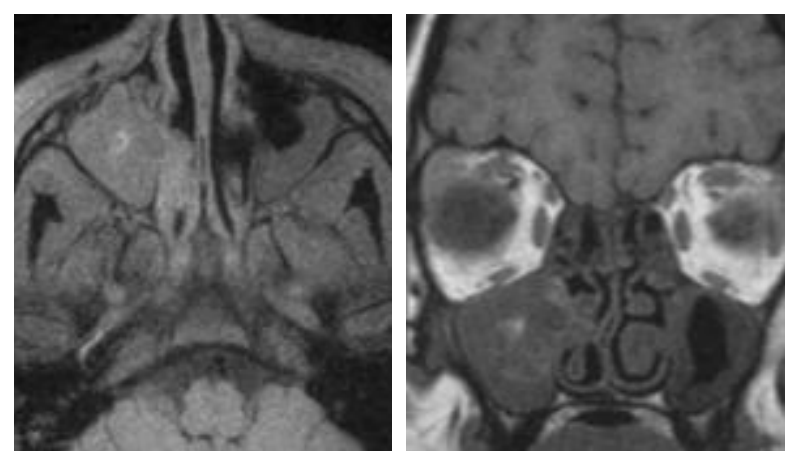

Fig 2: IRM en T1 en coupe axiale (a) et en coupe coronale avec saturation de graisse (b)

Opacité du sinus maxillaire droit hétérogène, siège de quelques foyers en hypersignal avec le même aspect sur les clichés avec saturation de graisse.
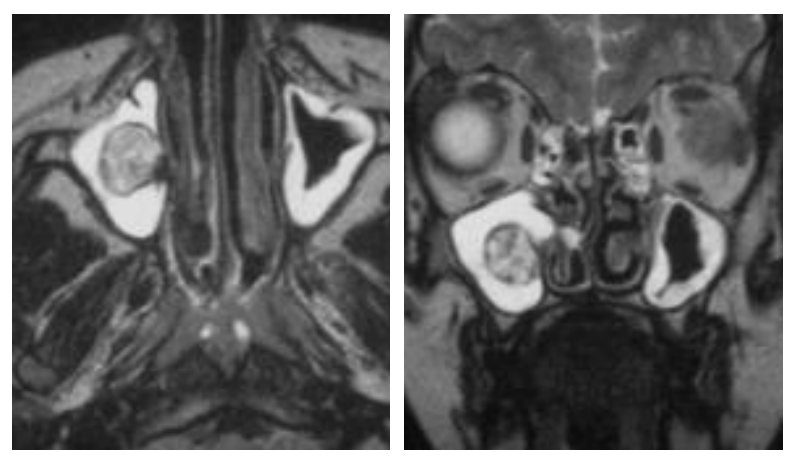

Fig 3: IRM en coupe axiale (a) et coronale (b) en T2.

Formation du sinus maxillaire droit hétérogène en hypersignal avec une hyperplasie muqueuse des deux sinus maxillaires.

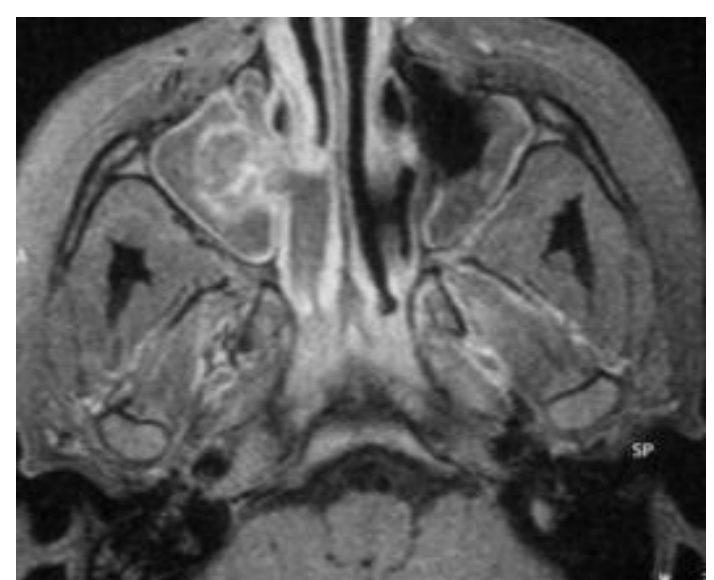

Fig 4: IRM en coupe axiale en T1 et avec injection de PDC. Formation du sinus maxillaire droit se rehaussant de façon hétérogène au sein d'une opacité totale du sinus.
Après rétablissement de l'état hémodynamique par des transfusions sanguines, la patiente a été opérée en urgence. Elle a eu une exérèse chirurgicale complète en monobloc par voie combinée : endoscopique et Caldwell Luc d'une formation de $2 \mathrm{~cm}$ polylobée encapsulée de couleur noirâtre. Le saignement per opératoire était minime et maîtrisable. Les suites étaient simples. L'examen anatomopathologique a révélé un hématome organisé du sinus maxillaire droit.

Nous n'avons pas observé de récidive après un recul de 4 mois. L'endoscopie nasale de contrôle était strictement normale.

\section{DISCUSSION}

L' HOSM a été décrit pour la première fois au Japon en 1917. Durant les 10 dernières années, 21 cas ont été rapporté dans la littérature anglo-saxone $(1,2,3)$.

L'étiopathogénie de l'hématome organisé du sinus maxillaire reste incertaine.

L'hématome du sinus maxillaire évolue sur plusieurs étapes. Initialement le sang stagne au niveau du sinus à cause d'une pauvre ventilation et de mauvaises conditions de drainage. Par la suite une couche fibreuse contenant des néo vaisseaux se forme tout autour, empêchant la résorption de l'hématome (4).

La progression du volume de l'hématome est due à un gradient de pression osmotique entretenu par l'hémorragie régulière des vaisseaux périphériques de cette masse au niveau de la capsule se rompant au fur et à mesure que cette masse se distend.

La cause de l'hémorragie initiale est variable : un traumatisme facial, un trouble de l'hémostase, une hémorragie post chirurgicale (4). Cette cause reste indéterminée dans la plupart des cas publiés $(5,6)$, comme c'est le cas dans notre observation.

Cliniquement l'hématome organisé du sinus maxillaire se manifeste par des épistaxis récidivantes, une obstruction nasale, des algies faciales, une tuméfaction jugale et rarement par une exophtalmie. La survenue de l'hématome organisé dans d'autres sinus outre le sinus maxillaire n’a pas été rapportée dans la littérature.

La tomodensitométrie avec injection de produit de contraste permet de préciser le siège et l'aspect de la lésion et de faire un bilan d'extension local et régional. L'aspect est celui d'une masse tumorale du sinus maxillaire, spontanément hyperdense hétérogène, prenant le produit de contraste de façon hétérogène avec fréquemment une lyse osseuse de la paroi médiale du sinus maxillaire. Une extension vers le sinus ethmoïdal homolatéral reste rare.

Le scanner est également très utile au choix du procédé thérapeutique le mieux adapté (voie d'abord, prévision sur les difficultés d'extraction).

Les diagnostics différentiels cliniques et radiologiques de l'HOSM se posent avec la mucocèle, le papillome inver- 
sé, la balle fongique, l'hémangiome, et le carcinome du sinus maxillaire.

La confirmation diagnostique reste histologique. L'examen anatomopathologique d'un HOSM montre de la fibrose, des néo vaisseaux et des globules rouges extravasés sans signes de malignité.

Le traitement de l'hématome organisé du sinus maxillaire est exclusivement chirurgical. Une évacuation complète est nécessaire pour assurer une guérison définitive. Plusieurs voies d'abord ont été décrites dans la littérature : la voie endoscopique endonasale seule (7), la voie vestibulaire (4), la voie combinée associant les deux ; la voie para latéronasale et même la voie de Rouge Denker (2) ont été décrites en cas de très forte suspicion d'une pathologie maligne. Le choix de l'une ou l'autre voie dépend de l'étendue de la lésion, mais la voie combinée reste la voie la plus souvent utilisée.

Le pronostic est généralement bon et les récidives sont très rares.

\section{CONCLUSION}

Bien que rare, le diagnostic de l'HOSM doit être évoqué devant une lésion évoquant une tumeur unilatérale du sinus maxillaire avec une épistaxis récidivante; même en l'absence de notion de traumatisme ou de trouble de l'hémostase, ceci permet d'éviter une chirurgie mutilante pour une pathologie bénigne.

L'aspect radiologique n'est pas spécifique.

L'exérèse chirurgicale doit être complète. Elle permet d'assurer est un meilleur pronostic pour le patient.

\section{REFERENCES}

1.Lee BJ, Park HJ, Heo SC. Organized hematoma of maxillary sinus. Acta Otolaryngol 2003; 123:869-72.

2.Yagisawa M, Ishitoya J, Tsukuda M. Hematoma-like mass of the maxillary sinus. Acta Otolaryngol 2006; 126:277-81.

3. Yoon TM, Kim JH, Cho YB. Three cases of organized hematoma of the maxillary sinus. Eur Arch Otorhinolyngol 2006; 263:823-6.

4. Tabaee A, Kacker A. Hematoma of the maxillary sinus presenting as a mass a case report and review of literature. Int J Pediatr Otorhinolarygol 2002; 65:1537.
5.Ho Kyu Lee, Wendy R, Smoker k. Organized hematoma of the maxillary sinus: CT findings. American Journal of Roentgenology 2007; 188:370-3.

6.Hyung Min Song, MD, Yong Ju Jang. Organizing hematoma of the maxillary sinus. Otolaryngology Head and Neck Surgery 2007; 136:616-20.

7.Yoon TM, Kim JH, Cho YB. Three cases of organized hematoma of the maxillary sinus. Eur Arch Otorhinolaryngol 2006; 263:823-6. 